\title{
Téoros
}

Revue de recherche en tourisme

\section{Éducation sur mesure « Entrepreneurship récréo-touristique " : une formation à la hauteur des régions du Québec et de leur environnement particulier}

Jean-Claude Jay-Rayon et Brigitte Morneau

Volume 11, numéro 1, mars 1992

La formation en tourisme : à la croisée des chemins

URI : https://id.erudit.org/iderudit/1078947ar

DOI : https://doi.org/10.7202/1078947ar

Aller au sommaire du numéro

Éditeur(s)

Université du Québec à Montréal

ISSN

0712-8657 (imprimé)

1923-2705 (numérique)

Découvrir la revue

Citer cet article

Jay-Rayon, J.-C. \& Morneau, B. (1992). Éducation sur mesure "Entrepreneurship récréo-touristique " : une formation à la hauteur des régions du Québec et de leur environnement particulier. Téoros, 11(1), 29-33. https://doi.org/10.7202/1078947ar 


\section{Éducation sur mesure «Entrepreneurship récréo-touristique") : une formation à la hauteur des régions du Québec et de leur environnement particulier}

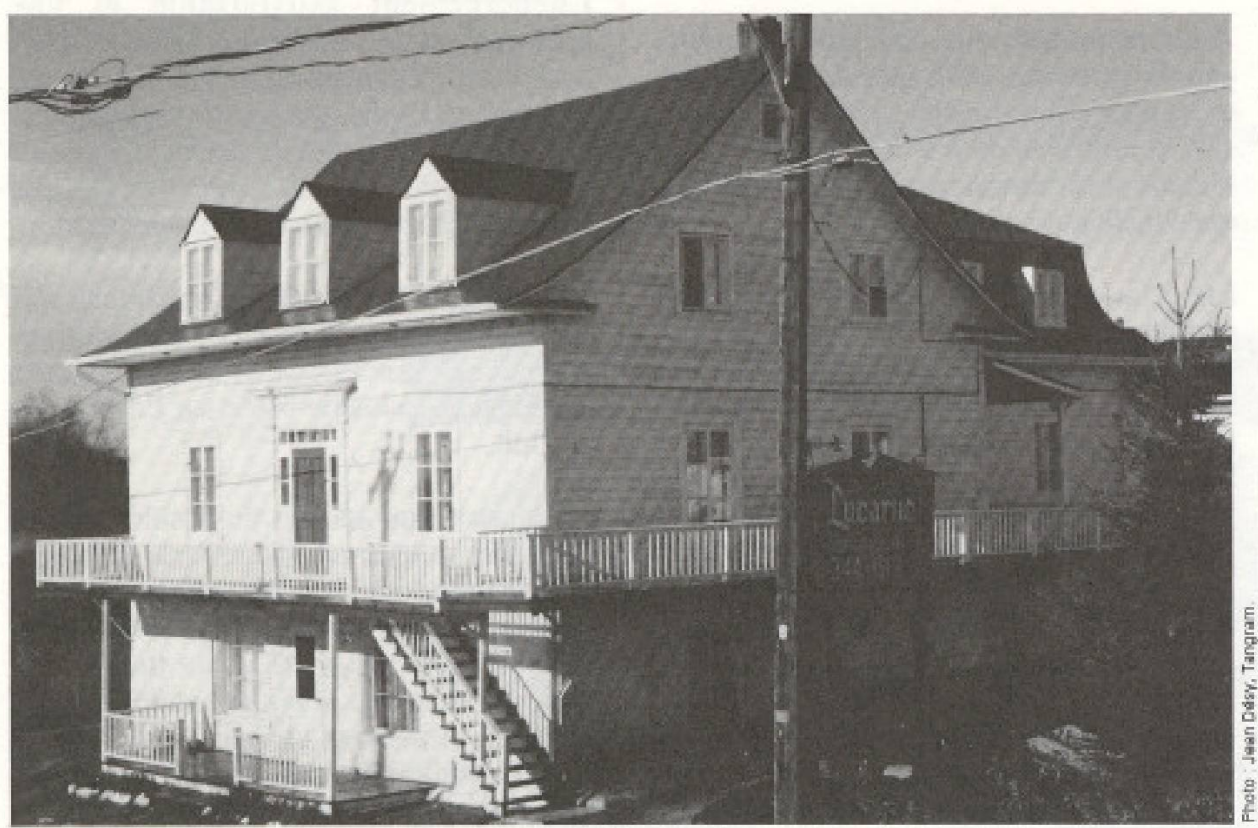

Auberge La Lacume. Vilage dHeberwille, quadrilarere urbain patrinonial, paroiso-mère du Lae \$t-Jewn.

Depuis quelques années, une formation sur mesure continue est venue, à titre encore expérimental, completer les nombreuses formesd'education au recréo-tourisme déjàdisponible.

La raison principale d'une telle démarche pédagogique était de répondre, surtout en région, à un besoin de connaissances bien précis qui touche les localités qui veulent redévelopper sur des bases originaleset économiques leur environnement à la fois naturel, semi-naturel et culturel.

Pour ce faire, un processus d'apprentissage assez strict et évolutif et des contenus élaborés pour répondre aux besoins très particuliers de chaque milieu concerné ontété conçu sur une base a la fois scientifique et technique. Ce qui amène l'étudiantà redécouvrir son environnement et à en extraire des qualités spécifiques, à le mettre en valeur et à le transformer pour qu'il devienne

\footnotetext{
Monsieur Jean-Claude Jay-Rayon est géo aménagiste et concepteur, chargé de cours a la maitrise ATDF de I'Universite Laval, Madame Brigitte Morneau est éducatrice, récréologue, associée de la firme EcartTangram de Lovis.
}

6conomiquement viable et enfin, à générer une très petite entreprise à but lucratif.

Jusqu'à présent, les résultats apparaissent suffisamment intéressants pour que certaines lignes de force puissent être dégagées des expérimentations passés et de cellesencours!

En premier lieu, on peut constater qu' il n'y a pas nécessité d'engager de nouveaux fonds pour la formation mais qu'il s'agit plutôt d' utiliser ceux déjà disponibles dans les CFP et ce, à des fins plus spécifiques et continues. Autrement dit, améliorer les formations courtes de quelques heures et les réintroduire dans un processus pédagogique cohérent de durée variant entre 300 et 600 heures maximum.

Deuxièmement, il apparaît nécessaire que les contenus de fommation soient baš́s sur des amodeles prócis performants et dejà existants donnant ainsią l'étudiant une réelle connaissance pratique des éléments à maîtriser et une assuranoe quant à la validité de ses apprentissages.

De plus, il semble a priori indispensable que la formation face en sorte de ramener l'étudiant sur une base qui englobera a la fois le milieu naturel et culturel, la récréation, le tourisme et l'6conomie. Ce qui exige en général de resituer les domaines de $l^{\dagger}$ hébergement, de la restauration et de l'accueil à un niveau secondaire.

Enfin, il apparaît que la formation sur mesure en région pour les adultes n'a de chance de réussir que si elle est large, systémique, efficace et personnalisée à la fois. Elle doit procurer à l'étudiant une plate-forme de connaissances très solides, utiles, donc signifiantes et nouvelles, sur laquelle il s'appuiera pour créer son entreprise. Les réflexions que font actuellement les intéressés quant aux formations en récré-tourisme, c'est que, d'une part, dans l'ensemble, elles correspondent surtout à une demande et à une offre touristique urbaine. D'autre part, elles sont trop longues et générales ou encore purement technique et restreinte. Devant une telle situation perçu, il devient alors indispensable de poursuivre la réflexion non seulement au niveau des techniques jusqu'alors enseignées, en allant beaucoup plus loin dans la rénovation des contenus actuels, mais aussi, de reconsidérer la formation sur mesure elle-même. Ni l'une ni l'autre ne semblent être tout à fait prêtes à répondre à un niveau de créativité et d'originalité que le produit touristique exige de plus en plus en région pour se maintenir dans un marché très exigeant et concurrentiel.

Mais en même temps, et en accord avec l'objectif de développer les régions,

... il importe de créter des entreprises pouvant apporter sur le marché de nouveaux biens ou services et de susciter, pour les ressources humaines, de nouvelles opportunités d'emplois. Cependant, cette recherchede creation d'entreprises etd'emplois devrase faire dans un souci constant de conserver la qualité de l'environnement(1).

Au cours des demières annés et ceci sans doute par nécessité, $l^{\prime}$ intérêt s s'est porté de plus en plus sur le faitd'inciter des personnes à démarrér des entreprises récréto-touristiques à la fois originales etrentables, dans leur propre milieu de vie. Il $s^{\prime}$ agit donc maintenant de former de futurs entrepreneurs capables une fois les cours terminés et, dans un délai raisonnable d'un an environ, de lancer 
ou de parfaire une très petiteentreprise (TPE) avec une mise de fond minimale et une garantie de réussite acceptable.

Nous disons que c'est d'abord une nécessité qui prévaut à l'élaboration d'une pédagogie particulière en récréo-tourisme car, il faut le reconnaître, si l'on trouvait sur place, en région, des gens déjà adéquatement éduqués et prêts à s' intégrer dans un schéma de développement, aménagement de type $\mathrm{PCL}(2)$ par exemple, il n'y aurait alors qu'à les recommander à l'emploi des municipalites ou MRC concernées.

Or, dans la plupart des cas les personnes disponibles sont des individus spécialisés en activités de plein-air pour clubs d'initiés, en loisir municipal, en sciences de la nature, en accueil pour kiosque routier, en écologie, etc., mais peu sont capables de prendre en charge une entreprise en se servant de la mise en valeur spécifique de son propre environnement naturel et culturel sous-régional ou local.

Nous faisons donc face, d'une part, à des gens trop sectoriellement spécialisés en canot, pecche à la mouche, escalade, interprétation, etc. Et, d'autre part, à des individus formés comme généralistes en tourismemais difficilementintégrablesà un environnement global et précis à la fois, comme par exemple, la Baie des Chaleurs en Gaspésie, la CôteNord, le Lac Saint-Jean ou la ville de MontSaint-Hilaire.

C'est d'ailleurs cet état de situation très concret qui a provoqué l'intérêt et suscité une curiosité au point de devoir wcréer sur mesure $\cdots$ une approche pédagogique efficace, à mi-chemin entre la formation très étendue et technique des Cégeps et celle, sur mesure mais, à la pièce, très répandue et financée par Emploi et Immigration Canada et gérée par les CFP.

Ainsi furent conçues et expérimentées avec des résultats différents mais sur une base résolument nouvelle etefficiente, 780 heures de cours en Gaspésie, 460 heures en BasseCôte-Nord, 200, 400 et 800 heures au Lac Saint-Jean et 600 heures pour la ville de Mont-Saint-Hilaire. Tout ceci au coút global d'environ 300000 \$attribuéa la seule formation de ce type.

\section{L'environnement naturel et culturel régional comme fondement de la formation sur mesure}

Comme nous l'avons mentionné, ce sont les schémas structurants d'aménagement et dé- veloppement de type PCL alors développés dans le cadre des sommets 6́conomiques et qui avaient pour but de remettre en valeur les caractếristiques de l'environnement culturel et naturel local, qui ont déclenché la quasi obligationd'obtenirune compétencehumaine correspondant aux lieux mêmes d'exploitation.

Aussi surprenant que cela puisse paraître, peu de personnes sont spécifiquement formées pour s'inscrire avec une grande précision et efficacité dans le milieu physique et humain et en faire une exploitation économique viable pour eux.

Les gens actuellement disponibles le sont surtout pour répondre à des besoins de service ou comme employés dans les secteurs classiques de l'hébergement, de la restauration, de l'accueil routier existants et plus ou moins dejà satures. Mais très peu sont formés pour créer une entreprise originale qui compléterait leproduitrégionaldéjàenplace. Il y a en somme un surplus de personnel à employer et une dramatique pénurie de produits nouveaux, eux-mêmes potentiellement employeurs de leurs createurs. En d'autres termes, l'éducation sur mesure devrait plutôt se situer dans le champ de la créativité que dans celui de la gestion ou du simple marketing. A cet effet d'ailleurs, rien n'est pire pour une région ou une localité que d'augmenter sa publicité, alors que son produit s'affaiblit ou encore, est de moins en moins significatif.

Le deuxième manqueflagrant que nous avons pu constater chez l'étudiant c'est une perte des connaissances fines reliées aux caracteristiques esthétiques et évocatrices du lieu d'exploitation.

En un sens la réussite touristique, comme le disait déjà René Dubos il y a vingtans, repose sur la connaissance approfondie et perceptuelle, voire sensorielle de l'environnement. Or, très souvent on ne connaît plus son propre milieu physique. On en a en quelque sorte perdu la mémoire et on ne voit plus la spécificité ou l'élément de curiosité a exploiter pour le touriste ou plus simplement, à signaler à partir de la route!

A cet effet, la formation nouvelle qui est foumie pourrait en partie combler ce vacuum. A notre avis, l'étudiant devrait acquérir une connaissance particulière concernant la geographie perceptuelle de son milieu et des comportements humains qui en découlent bien avant d'aborder des techniques de locomotion, de mise en marché, de gestion, de comptabilité, etc.
Concernantcetaspoctenvironnemental, nous nous sommes aperçus dans différentes sousrégions que, quelquefois, $60 \%$ à $80 \%$ des endroits à développer étaient ignorés par le futur entrepreneur. Comment, devant une telle méconnaissance de base, penser alors une signalisation adéquate, une architecture harmonieuse et intégrée, une orientation de l'hébergement satisfaisante et une implantation de l'entreprise à l'endroit exact? Rien d'etonnant alors de constater que trèss souvent l'infrastructure touristiquen'est pas au bon endroit, à commencer par certains kiosques d'information et que de ce fait, elle ne peut être correctement «rentabilisée».

D'ailleurs, le ministère du Tourisme dévoilait récemment sa nouvelle politique en reconnaissant la nécessité de doter le Québec d'une réelle industrie basée sur l'approcheproduit.

L'industrie doit adopter un positionnement distinctif, unique et clair en vue de bâtir à moyen terme une véritable personnalité touristique qui la démarque de ses concurrents. $\mathrm{Or}$, la démarche passe avant tout par un choix. Le couple wculture-natures, fondement des principaux attributs du Québec sur le plan géo-touristique, est retenu comme positionnement générique(3).

\section{Éduquer à la culture. Oui, laquelle?}

Dans le sillage du rapport Arpin sur la culture et à la suite de la récente politique sur le tourismequipositionnedorénavant leQuébec en associant dans un même concept natureculture, les étudiants $d^{+}$une formation sur mesure continue se devraient d'aborder le produit culturel suivant des angles originaux et novateurs.

Tout d'abord, le futur entrepreneur devrait pendant sa formation plus ou moins apprendre qu"en matière touristique le domaine culturel ne se limite plus à mettre en valeur le seul passé patrimonial, historique ou folklorique d'une région pour créer un attrait significatif et donc attractif de qualité.

Il est ainsi peu probable qu'une majorité de régions puissent constituer un produit culturel pouvant rivaliser avec celui des villes et cela dans les mêmes domaines muséologiques, artistiques et patrimoniaux.

En un certain sens, les régions comprennent très bien les limites d'une démarche compétitive face aux pôles de Montréal et Québec et elles sont à même de se toumer résolument vers des représentations culturel- 
les inusitées, surprenantes, et majeures, ou l'originalité du produit sera à toute épreuve. Nous entendons par là toute la différence qu'il peut exister entre une pièce de theâtre produite au Rideau vert à Montreal et l'Histoire d'un Royaume présentée à la Baie, au Saguenay.

A cet effet, on peut penser que les représentations touristiquesculturelles traditionnelles pourraient aisément s"enrichir de nouveawx courants de pensée et de réalisation visant a mettre en valeur les qualités du vaste environnementdisponibleàl'extérieur des villes, donc, au niveau régional et local.

C'est dans ce contexte que les étudiants formés dans le cadre pédagogique qui nous intéresse iciontếté introduits à une démarche culturellequi fait résolument référence al' art d'artisanat et à l'économusćologie telle que definieparCyril Simard, àl'éconoculture, au Land art, au Earthworks, au macro-art pictural de type Chemainus, etc. Autant d'exemples concrets qui devraient permettre a l'étudiant de se servir de son environnement disponible, de son village, de ses champs, voire de son agriculture, de ses carrieres de pierre industrielle éventuellement, pour fabriquer un produit culturel hautement original et rentable, LA, comme en ce qui a trait à l'environnement naturel, l'environnement culturel est enseigné dans l'optique d'une culture du futur performante, capable de participer à l'économie locale et qui devrait ainsi compléter l'approche passéiste déjà largement exploitée.

\section{Éduquer à l'économie environnementale : un nouvel apprentissage indispensable}

Dans de très nombreux cas, il est à remarquer que l'étudiant se situe face à la rentabilité de sa future entreprise dans une situation paradoxale. Il y a par exemple confusion chez lui entre le fait de créer son affaire, donner une plus value à un territoire ou à un bâtiment et en vivre honorablement et une certaine mentalité de salarié à l'emploi d'une entreprise récréo-touristique quelconque.

D'un côté, il s'agit d'acquérir des connaissances très completes pour que l'entreprise non seulement soit viable mais fasse des bénéfices et progresse dans un marché déjà encombré. De l'autre, il ne faut qu'apprendre à être un excellent employé dans certains domaines spécifiques comme la restauration, le marketing, l'accueil, etc.

Or, un futur entrepreneur régional ou local n'est pas d"abord un employé dans le con- texte actuel. S'il veut réussir, il lui faudra apprendre commentcréer un produit de haute qualité, originaletconcurrentiel, avant d'être un individu aux compétences techniques ou ouvrières. Et c'est à ce niveau que certaines connaissances associant 6 cologie, 6 conomie et culture sont indispensables à obtenir sous peine de ne jamais être capable de bâtir une entreprise rentable.

Unedes principales faiblesses que lapédagogie se devrait de corriger est celle qui réside dans une certaine timidité à exploiter de façon commerciale et financière un environnement touristique de qualite.

En général, l'étudiant est pris entre deux notions que la société lui présentent idéologiquement, comme irréconciliables: la gratuité collective des gisements environnementaux naturels et culturels et la mise en valeur de ces mêmes environnements à des fins d'industrie touristique et privece mais, non exclusive.

Ils'agit làd' un véritable dilemme au Québec, dont il faut que l'étudiant $s$ "extraie avec des connaissances adéquates car son entreprise récréo-touristique ne pourra bien exister que si elle se situe au coeur même d'un environnement optimum.

En un certain sens, la réussite d'une formation à l'entrepreneurship en récréo-tourisme ne peut passer outre au fait de cette notion de valeur ajoutée et d'exploitation des milieux naturels et culturels. Sans cette approche fondamentaleetintégrée, l'étudiant nepourra jamais placer son entreprise ou son projet la où il devrait ĉtre, ni non plus mettre en marché sous de multiples formes les caractéristiques d'un milieu donné.

II sera toujours rejeté ou situé a la périphérie d'un parc, d'une réserve, d'un bâtiment patrimonial, d'un site significatif, etc. En somme toute approche éducative récréo-touristique devrait contenir des apprentissages mettant en valeur l'economie environnementale, base même du produit touristique moderneetà parfaire ou à bâtir dans plusieurs régions et localités.

\section{Quatre exemples de très petites entreprises (TPE) réussies}

On ne peut évaluer l'efficacité d'un enseignement voué au démarrage de la très petite entreprise récréo-touristique à but lucratif dans une région sans en présenter quelques success story.
Ceux qui suivent originent d'un cours d'à peine 200 heures qui fut suivi d'un autre d'environ 800 heures et qui furent donnés au LacSaint-Jean. Le financement provenait de laCFPet la démarche pédagogiqueétait sous la responsabilité de l'éducation des adultes du Cégep de Saint-Félicien et du coordonnateurde l'époque, monsieur Jacques Lefebvre.

En somme, toute la démarche en ctait une de concertation entre la commission de formation professionnelle, la maison d'education et une firme privée apte à élaborer une démarche et un contenu educatif sur mesure, collant a la fois au potentiel environnemental régional et aux attentes des futurs étudiants et à leur capacité créatrice réelle.

Ce qu'il faut aussi ajouter, $c^{\prime}$ est que chaque étudiant possédait déjà, avant le cours, un projet d'entreprise, voire une entreprise, et donc une anticipation plus ou moins bien definie. Une sorte de projet concret qu'il acceptaitd'ajuster avecleséléments du cours, dans le but de l'enrichir ou de le modifier au fur et à mesure.

Ce détail est très important à mentionner car on ne peut donner un cours de démarrage d'entreprise récréo-touristique avec un pourcentage de réussite adéquat que si, au préalable, l'étudiant s'est au moins fait une idée convenable de ce qu'il veut entreprendre.

On ne vient pas dans une formation sur mesure d'un certain type avec l'idée un peu naive $d^{*}$ avoir instantanément un job à la fin de celle-ci, ou une recette magique pour réussir en affaires, obtenir des prêts, etc.

Cela exige de la maison d'éducation un recrutement de candidats bien sélectionnés. Ce qui est tout le contraire d' une inscription d'étudiants quelques jours seulement avant le cours.

\section{Quant aux problématiques}

Les municipalités, corporations et organismes qui requièrent un certain type de formation sur mesure à l'entrepreneurship sont soumis à des problématiques de fond qui ne peuvent souvent être résolues qu'à moyen terme. C'est sans doute pour cette raison qu' une démarche pédagogique qui se situe entre la courte et la longue formation semble convenir et aboutir avec efficacité à des résultats probants et vếrifiables. En considérant des delais de 300 à 800 heures de cours, on peut ainsi agir avec l'étudiant au niveau fondamental et en même temps au plan très concret d"une entreprise à parfaire ou à créer. 
En somme, l'adulte a non seulement le temps de $s$ 'instruire mais aussi de concrétiser ses connaissances sur le terrain sans avoir $l^{\text {'im- }}$ pression de perdre du temps ou d'apprendre seulement à être l'employé de quelqu'un d'autre.

Pour plusde précision, ilest toutefois interessant de considérer les quelques problematiques qui ont sous-tendues la démarche exposee ici.

En 1988, sur la Baie des Chaleurs, les statistiques démontraient un besoin derenouvellement de l'industrie et d'adaptation de la main-d'oeuvre au plan touristique. Il etait précisé que:

... par dessus toute cette problématique du développement socio-économique, s'ajoute le constat de la non réussite de TPE et de PME; échec attribuable en grande partie, à une faible scolarité et à un manque flagrant de connaissances fondamentales au niveau de l'administration et de la gestion d'une entreprise, si petite soit-elle (page 13). L'implantation de services de tres petites entreprises récréo-touristiques entraînant une utilisation rationnelle et respectueuse du milieu, c'est-à-dire l'emploi des ressources naturelles et humaines de l'environnement, et cela, en vue d'augmenter et de compléter l'économie régionale de la Baie-des-Chaleurs.(4)

Pour la Basse-Cote-Nord en 1989, l"on se devait d'aller encore plus loin et de procéder à un transfert economique et technologique de l'industrie de la pêche à celle du récréotourisme.

Depuis quelques annees, les habitants de l'archipel de Harrington Harbour constatent que la ressource maritime diminue. Le passage graduel sur une période de cinq à six ans à une économie touristique complémentaire devient donc une hypothèse sérieuse à considérer. La formation, ence sens, devient ainsi une priorité pour permettre la mutation qui $s^{5}$ impose(5).

Pour la région du Lac Saint-Jean, la problématique s'articulait beaucoup plus autour du besoin de renouvellement du produit et des services offerts, a l'amelioration de la qualite des infrastructures facilitant l'acò̀s à l'environnement naturel et à l'ajout de sites originaux et attractifs.

Le Lac Saint-Jean est depuis plusieurs années une destination touristique reconnue, mais le produit n'a pas été renouvelé et la perception du rapport qualité/prix est néga- tive. Il manque d'activités bien intégrées au milieu et propres àaugmenter larétention des touristes(6).

Quant à la municipalité du Mont-SaintHilaire, celle-ci a choisi d'associer, dès le départ, les propositions de formation aux recommandations de développement. La réalisation d"un plan d ${ }^{7}$ Education sur mesure asuivi le dépôt de l'étude PCL et le processus d'audiences publiques.

Mont-Saint-Hilaire se voitconfronté àcourte échéance à deux phénomènes majeurs: l'un qui concerne son redéveloppement 6conomique au niveau récréc-touristique, l'autre qui consiste en l'obligation de former des gens compétents pour assumer cette démarche. Meme si les gens souhaitent se diriger vers unéeconomie récréo-touristique, il leur est difficile de formuler de façon pratique des projets rentableset novateurs(7).

\section{Les exemples}

\section{Le cas de l'économusée}

"Sensation Nature"

Monsieur Régis Gauthier ne possédait aucun emploi mais il avait pu expérimenter quelques années auparavant une idée d'exposition faunique et taxidermique dans le cadre d'un festival populaire. La curiosité de la clientèle avait été telle que cela l'encourageaitàmettresur pied unesorte d'économusée au Lac Saint-Jean. Le cours lui a permis de modifier son projet initial, de le rendre mobile et itinerant, de s'inspirer des décors de cinéma et de bâtir son projet sur une base scientifique à caractère éthologique et écologique. En un peu plus d'un an, cette personne $s^{*}$ associa à un homme d'affaires, il obtint des crédits gouvernementaux, construisit un produit original de $1 / 3$ de million de dollars pouvant employer 10 individus et se trouve ainsi prêt des le printemps 1992 à être opérationnel sur l'ensemble de la province. SaTPEsenomme Sensationnature etlecours d'entreprenariat lui a, de ses propres dires, octroyé $50 \%$ de connaissances nouvelles et originales sans lesquelles son entreprise n'aurait pu voir le jour. Actuellement, deux contrats majeurs sont déjà en sa possession pour l' annee 1992.

\section{Le cas du "Centre Vacances Hébertville"}

Madame Nicole Hudon était au début de son cours échevin du village d'Hébertville et suite au sommet économique; elle avait été nommée responsable du tourisme pour son agglomération. Son objectif était donc d'obtenir une fomation générale dans un do- maine qui lui ctait presque inconnu. Au fur et àmesure du cours, son information se mua enconnaissances assez précises pour luidonner le goût de démarrer sa propre entreprise et de lancer son agence réceptive. De sa propre appréciation, la formation lui apporta $90 \%$ de nouvelles connaissances sur l"environnement de son village et sur le potentiel qu' ilrenfermait. En troisans, madame Hudon, non seulement fonda sa TPE originale mais dirigea une progression fulgurante de son entreprise.

En 1989-1990, dès la première année d'opération, elle reçue en hébergement famille, 12 groupes d'Européens. En 1990-1991, à sa deuxième année, la clientèle passaà 16 groupes et elle pu prendre de l'expansion en ajoutant des services comme la location de chalets, les forfaits d'eté et d'hiver. En 19911992, dès la troisième année, 32 groupes furent reçus, plus 275 personnes individuelles, pour un total de 2650 nuitées! Déjā pour 1992-1993,54 groupes ont réservé pour un total de 4000 nuités. Quant au chiffre d'affaires, celui-ci est passé de 19146 \$ la première anné à $89100 \mathrm{~S}$ en 1991. Ce qu'il faut noter au passage, c'est que dans ce cas précis, la TPE de madame Hudon a réussi dans un village qui n' était même pas reconnu dans le circuit touristique classique, malgré son fort potentiel patrimonial, historique et esthétique.

\section{Le cas de I"Auberge La Lucarne"}

Avant de suivre son cours, madame Paule Jean possédait une idé et une volonté précise: transformer la maison familiale où avait ćtéçlevéses enfantsen un type d'auberge ou de Bedand Breakfast. Cette bâtisse, d'une belle architecture et possédant au-dela de 20 pièces était située en plein centre du village d'Hebertville, dans ce qu'il est convenu de dénommer le quadrilatêre patrimonial et datait de 1860. En 1972, madame Paule Jean et son mari en avait fait l'acquisition pour 12 250 S et au fur et à mesure des annóes, ils avaient ajoutéenviron $50000 \$$ de travaux de rénovation. En 1989, madame Paule Jean suit donc le cours d'entrepreneurship récréotouristique etavantmême de l'achever, lance son Auberge de la lucame avec 4 chambres seulement. Plus tard, elle en ajouta 4 autres. Des 1990 , elle reçit 140 personnes et en 1991, 305; déjà pour 1992, 180 ont réservé. Elle effectue donc en un an une progression de $100 \%$ et samaison est dorénavantestimé aux environs de $200000 \$$. Phénomènes. intéressants à noter: la non plus le village d'Hébertville n"apparaissait pas dans le circuit officiel, la clientèle de l'Auberge de la lucarne est étrangère et quelqu' un d'officiel 
déconseilla même à madame Paule Jean de se lancer dans une telle affaire à moins d'investir au minimum 20000 \$ par chambre! Comme quoi on peut, à partir de certaines connaissances apprises, faire beaucoup mieux, pour moins cher et sans subvention gouvernementale! D'ailleurs, dans ce cas précis, si la formation n'avait pas enrichi madame Paule Jean de $40 \%$ a $50 \%$ de connaissances nouvelles et exactes, les conseils en développement qu' elle avait reçuel'aurait instantanément conduite à un investissement démesuré, non amortissable. C'est-à-dire à la faillite pure et simple!

\section{Le cas de l' Auberge de la petite chute"}

En 1989, madame Nicole Larouche était échevin d'une petite municipalité de 850 habitants situéc à environ $22 \mathrm{~km}$ de la route principale qui ceinture le Lac Saint-Jean. La dernière agglomération avant l'immensité de la forêt! Contrairement à la plupart des étudiants, madame Larouche ne possédait pas d'idée précise à développer. C'est le cours en entrepreneurship récréo-touristique conçu par madame Brigitte Momeau qui lui aalors servi, àau-delà de $80 \%$ d'aprèselle, de motivation en lui apportant des connaissancesprécises. Cequemadame Larouche amis surpiedc'est une auberge forestière, donc un produit totalement nouveau et inconnu dans la région à $10 \mathrm{~km}$ de Ste-Hedwidge, sur une piste non asphaltée et à partir d'un vieux batiment acquis pour la somme de 9000 \$. Ici, le choix du siteetl'approcheproxémique a joué un rôle déterminant quant à la réussite du produit. L'hypothese était que l'environnement choisi representait en fait une cle d'entrée et de circulation donnant accès à une ZEC possédant 140 chalets. A I'heure actuelle, $1^{*}$ auberge forestiere la petite chute, est ouverte depuis le 11 mai 1991 . Elle a reçu avec 6 chambres plus de 100 personnes pour dormir. Certaines venues pour une nuit $y$ restent trois soirs. Certaines fins de semaines on y sert jusqu'à 300 repas sans compter les brunchs très populaires. La clientèle touristique est interrégionale, américaine et européenne. L'auberge est maintenant évaluce à 300000 \$ et madame Larouche en est le chef d'entreprise. L'élément ici à noter, c'est qu' un produit peut émerger dans un environnement de haute qualité et réussir dans une région qui, elle, subit une diminution de touristes. Autre fait à mentionner, le village de Ste-Hedwidge ne faisait non plus pas partie des circuits touristiques officiels et encore moins l'endroit où est situé L'auberge de la petite chute qui emploie maintenant 6 personnes.

\section{Conclusion}

La formation sur mesure pour adultes dans le domaine récréo-touristique soulève une problématique toute particulière dont le premier critère en est un de qualité et de précision quant aux contenus enseignés. L'étudiant adulte n'a ni le temps, ni la patience de s'astreindre à un long processus théorique. De plus, il est en général suffisamment au courant des difficultés du marché du travail régional ou local pour, intuitivement ou par expérience, se méfier d'apprentissages qui n' auraient pour but que de l'occuper temporairement.

En ce qui conceme le recrutement des étudiants adultes pour une formation sur mesure, cette demiere ne semble pas correspondre toujours au profil de clientèle souhaitce. Les normes de recrutement font en sorte d'accepter des gens parfois inaptes à fonder une entreprise quelconque ou même à se trouver un emploi dans le domaine du tourisme. Certains étudiants suivent des cours pour en suivreet ainsi sauver leurs avantages sociaux et financiers qui y sont rattaches. Dans le cas des expériences entreprises, il a ểeremarquéque les cas de réussiteoud "échec peuvent fluctuer de $10 \%$ $\$ 60 \%$ dependant de qui est accepté et des raisons pour lesquelles on suit un cours. A cela s'ajoute le travail de la maison d'éducation qui, elle, dit considérer ce type de formation avec sérieux par rapport aux autres elèves réguliers.

Quant à la formation elle-même, celle-ci doit tenir compte d'un ensemble de facteurs qui doiventêtre rassemblés au sein d"une démarche pédagogique cohérente. Il ne sert à rien d'enseigner l'une après l'autre des matières comme la comptabilité, le marketing, la gestion, $l^{\prime \prime}$ accueil, le paysage, l'artisanat, etc. à partir d'un rassemblement hétéroclite d'enseignants et de contenus. Il faut à la formation sur mesure un tronc commun sûr et solide sur lequel l'étudiant pourras' appuyer. Dans un tel contexte, les CFP auraient peutêtre alors awantage à réduire les sommes attribuées aux cours de courte durée et à encourager une formation plus étoffée et efficace.

Enfïn, et c'est là peut-ềtre l'elćment le plus délicat à maitriser, il faudraitque les matières enseignées en tourisme soient actualisćes et que l'étudiant puisseen appliquer des principes en equilibre avec sa région et sa localité propre. Il est tout à fait dérisoire de former des individus en leur apprenant ce que sont les chutes Niagara si ceux-ci ne sont pas capables de traduire cette culture générale en éléments pratiques dans leur région et dans le cadre exact de leur environnement. Il en est de même des montagnes ou chacun croit qu'il en possède une pour faire une station de ski touristique viable, des lacs que $1^{4}$ on imagine être des méditerranées ou des océans et des zoos qui ont du mal a saisir que le temps des animaux enfermés est en partic revolu. En bref, ce que l'adulte souhaite aborder dans sa fomation c'est un champ de connaissances objectives et vérifiables.

\section{BIBLIOGRAPHIE}

CONFERENCE BOARD DU CANADA, Institut Canadion de recherche en tourisme, Conférence wur lot perepectives touristiques du Oubbec, 1992, 98 p.

DUKAKIS, S, et ALDEN, S. Faine, Creating the Future: Opportunity. Innovation and Growth in the Massachusette Economy, Office of Economic Development, 1987, $82 \mathrm{p}$.

JAY-RAYON, Jean-Claude, Tendance: raí du large, Summum, vol. 2, no $3,1969, \mathrm{pp} .7-10$.

JAY-RAYON, Jean-Claude, Tendence: changer d'dre, Summum, wol. 2, no 6, 1990, pp. 6-22.

GOUNERNEMENT DU QUEBEC, ministêre du Tourisme, Énoncb de politique et plan d'action en matilire de tourleme, 1992, $96 \mathrm{p}$.

MOANEAU, Brigitte et COTION, Pierre, Initlation au dómarrage ot d la gestion de très petites entreprises TPE rocrío-touristiques. College de la Gasposie et des lles, Gaspé, 1988, 94 p.

MORNEAU, Brigitte, Initiation au prd-domarrage et

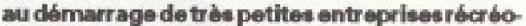
tourlstiques, Administration de la Basse-CoteNord et du Golfe Sain t-Laurent, Chevery. 1989, 26 p.

MOFNEAU,Brigitte, Entreprenariatet gention detrb: petitee entreprleses (TPE) en rdado-tourieme. Cégep de Saint-Félicien, $1989,32 \mathrm{p}$.

MORNEAU, Brigitte. Recyclage et perfectionnement de la main-d'oeuvre en rétubo-tourisme, Corporation touristique du Mont-Saint-Hilaire, 1991, $25 \mathrm{p}$.

QUEBEC, A theure de Fentreprise rogionale: plan d'action en matikre de díveloppement rbgional, OPDQ, $1988,90 \mathrm{p}$.

\section{NOTES EXPUICATIVES}

(1) Quebec, A Theure de T'entreprise réglonale, OPDQ. 1988, p. 20.

(2) PCL: Planification - Conception et Locallsation basées sur un environnement précis, de façon a a procurer au tounste la Perception et la Connaissance du milieu maximale pour une Locomotion minimale et efficace.

13) Québec, Énoncé de politique et plan d'action en matibre de touriame, MTO. 1992, p. XII.

(4) Brigitte Morneau, Initlative au dbmarrage ot a la gestion de trat petites entreprises récrbotouristiques, Cégep de Gaspé, p. 1.

(5) Brigitte Morneau, Initiation au pró-démarrage of au domarrago de trat potites entreprines récró-touristiques, Basse-Cóle-Nord, p. 5 .

(6) Brigitte Mornesu, Entreprenariat ot goation de tros petites entrepriees recrob-touristiques; C6gep de Saint-Felicien, p. 15.

(7) Brigitte Morneau, Recyelage et perfectionnement de la main-d oeuvre, Mont-Saint-Hilaire, p. 18. 